Machine Learning 2: 371-396, 1988

(C) 1988 Kluwer Academic Publishers, Boston - Manufactured in The Netherlands

\title{
Criteria for Polynomial-Time (Conceptual) Clustering
}

LEONARD PITT

ROBERT E. REINKE

Department of Computer Science, University of Illinois at Urbana-Champaign, 1304 West Springfield Avenue, Urbana, IL 61801, U.S.A.

(Received: September 1, 1987)

(Revised: January 9, 1988)

Keywords: Cluster analysis, conceptual clustering, analysis of algorithms
(PITT@A.CS.UIUC.EDU)

(REINKE@UICSL.CSL.UIUC.EDU)

\begin{abstract}
Research in cluster analysis has resulted in a large number of algorithms and similarity measurements for clustering scientific data. Machine learning researchers have published a number of methods for conceptual clustering, in which observations are grouped into clusters that have "good" descriptions in some language. In this paper we investigate the general properties that similarity metrics, objective functions, and concept description languages must have to guarantee that a (conceptual) clustering problem is polynomial-time solvable by a simple and widely used clustering technique, the agglomerative-hierarchical algorithm. We show that under fairly general conditions, the agglomerative-hierarchical method may be used to find an optimal solution in polynomial time.
\end{abstract}

\section{Introduction}

There is a wide body of literature in several fields concerned with the clustering problem. Roughly, this problem involves grouping observations into categories such that members of a category are alike in some interesting way and members of different categories are different. Within artificial intelligence and machine learning, the clustering problem has been classified as part of the general problem of learning from observation and discovery (Carbonell, Michalski, \& Mitchell, 1983).

Much of the work on the clustering problem has involved numerical or statistical techniques for clustering scientific data. Researchers in cluster analysis and numerical taxonomy have focussed on developing appropriate metrics for measuring similarity between points and clusters (groups of points), and on developing algorithms to minimize inter-cluster similarity as measured by some objective function. The literature on these techniques 
is scattered through journals in statistics, pattern recognition, computer science, and various fields of application (biology, psychology, sociology, etc.). Summaries may be found in Anderberg (1973), Hartigan (1975), Duda and Hart (1973), and more recently in Romesburg (1984).

In machine learning, work on the clustering problem has focussed on the notion of conceptual clustering, introduced by Michalski (1980). Conceptual clustering methods attempt not only to produce "good" classifications based on some metric, but also to find a meaningful description of the classification. In contrast, cluster analysis techniques leave it to the human analyst to determine the meaning of a clustering. ${ }^{1}$ Researchers in conceptual clustering have not only produced a number of algorithms and metrics (e.g., Lebowitz, 1983; Michalski \& Stepp, 1983; Fisher, 1985; Mogenson, 1987), but have also investigated employing clustering in problem solving (Rendell, 1983; Fisher, 1987), incorporating problem-specific knowledge into the clustering process (Mogenson, 1987; Stepp \& Michalski, 1986), and providing appropriate cluster description languages (Stepp, 1984; Fisher, 1985). Fisher and Langley (1985) and Stepp (1987b) provide overviews of work on conceptual clustering, and develop characterizations of the problem.

Instead of adding to the already large body of clustering algorithms and metrics, we have explored the properties of a simple, general, and wellknown clustering method - the agglomerative-hierarchical or amalgamative algorithm. In particular, we are interested in characterizing the kinds of problems, metrics, objective functions, and concept description languages on which a given algorithm will succeed. We define success as the discovery of an optimal clustering in time polynomial in the number of data points to be clustered. Though introductory texts on cluster analysis sometimes attempt to explain how to choose an appropriate algorithm and metric, to our knowledge there has not been any formal exploration of the conditions under which particular algorithms are guaranteed to produce optimal solutions in polynomial time. ${ }^{2}$ By specifying such conditions, we hope to simplify the problem of selecting an appropriate clustering technique.

In the next section, we introduce a simple conceptual clustering problem, and use it to motivate general definitions for clustering problems, distance metrics, and objective functions. In Section 3, we use the example to introduce the agglomerative algorithm and to motivate some fairly general restrictions on conceptual clustering problems. In Section 4, we prove

\footnotetext{
${ }^{1}$ Here, and throughout the remainder of the paper, we use the term "cluster analysis" to refer to all clustering techniques in which the quality of cluster descriptions is not a factor in measuring the quality of the clustering.

${ }^{2}$ Of course, there exist algorithms designed to produce an optimal solution for particular metrics and objective functions.
} 
these restrictions are sufficient to guarantee that the algorithm finds an optimal solution. We also examine further properties of the agglomerative algorithm, as well as variations of the restrictions of Section 3.

\section{Definitions}

We formally define a very general version of the clustering problem. The definitions are similar in spirit to the definition of the Abstract Clustering Task given by Fisher and Langley (1985). However, we want to more carefully specify what is meant by a "clustering quality function." We believe the definitions are general enough to subsume the objective functions normally used in cluster analysis and conceptual clustering.

We motivate the definitions, and the properties of the next section, with the following example of simplified conjunctive conceptual clustering (Michalski \& Stepp, 1983), or monomial clustering. ${ }^{3}$ For monomial clustering, the objects to be clustered are described with $n$ Boolean attributes $x_{1}, x_{2}, \ldots, x_{n}$. Let $X_{n}$ be the set of Boolean vectors over the attributes $x_{1}, x_{2}, \ldots, x_{n}$. Then the domain for the monomial clustering problem is $x=\left\{X_{n}\right\}_{n \geq 1}$.

Similarly, many other domains for clustering problems are best described as a parameterized family $\mathcal{X}=\left\{X_{n}\right\}$ of sets, where the parameter $n$ is some appropriate value, typically reflecting the "size" of an element. For example, if we are interested in clustering objects in Euclidean space, then the domain $\chi$ might be the collection $\left\{E_{n}\right\}_{n \geq 1}$, where $E_{n}$ is $n$-dimensional Euclidean space.

For some domains $\chi=\left\{X_{n}\right\}$, we may allow $X_{n}$ to be empty for some (or most, if no parameterization is desired) values of $n$. For example, if the only type of clustering problem that we wish to consider is clustering in 2-dimensional Euclidean space, then we would let $X_{2}=E_{2}$, and $X_{i}=\emptyset$ for $i \neq 2$. The above considerations motivate the following

Definition $1 A$ domain $\mathcal{X}$ is a parameterized family of sets $\left\{X_{n}\right\}_{n \geq 1}$.

Our definitions will require that a clustering algorithm work for all $X_{n}$ that are nonempty, and that the algorithm have run-time polynomial in $n$.

In conceptual clustering, a cluster is described by a statement in some language, and not by the set of points in the cluster. For example, for monomial clustering, clusters are described by monomials over $n$ Boolean attributes. Let $L_{n}$ be the set of all monomials (pure conjunctive concepts) over the Boolean variables $x_{1}, x_{2}, \ldots, x_{n}$.

\footnotetext{
${ }^{3}$ The formal definition of the monomial clustering problem will be given in Section 3.1.
} 
Definition $2 A$ clustering description language $\mathcal{L}$ is a parameterized family of languages $\left\{L_{n}\right\}_{n \geq 1}$.

The parameter $n$ typically reflects the size or length of statements in the language $L_{n}$.

Each statement $c \in L_{n}$ is a cluster. The meaning of a cluster is given by an interpretation:

Definition $3 A n$ interpretation $I=\left\{I_{n}\right\}_{n \geq 1}$ of a language $\mathcal{L}$ to a domain $\chi$ is a parameterized family of functions $I_{n}: L_{n} \rightarrow 2^{X_{n}} .\left(2^{X_{n}}\right.$ is the power set of $X_{n}$.)

For each $n$, every cluster $c \in L_{n}$ describes a set of points of $X_{n}$ given by $I_{n}(c)$.

For monomial clustering, the interpretation $I_{n}$ of a monomial over the variables $x_{1}, x_{2}, \ldots, x_{n}$ is the standard logical one, i.e., the set of Boolean vectors of length $n$ (over the same variables) that satisfy the monomial. For some applications, it is desirable to parameterize the family of languages $\mathcal{L}$ differently than the domain $\chi$. For example, if the goal is to cluster numerical data points based on their descriptions in a simple, limited language, then for each $n$ and $m$ there would be an interpretation $I_{n, m}: L_{n} \rightarrow 2^{X_{m}}$. For the sake of clarity, we will assume that the language and its domain have the same parameter $n$; the extensions required for the two parameter case are straightforward.

A clustering $C$ over $L_{n}$ is a finite set of statements (clusters) of $L_{n}$. The size of a clustering $C$ is the sum of the lengths of the statements in $C$. Let $K_{n}$ denote the class of all clusterings over $L_{n}$. For monomials, a clustering is simply a finite collection of monomials.

A cluster $c$ covers a set $S \subseteq X_{n}$ iff $S \subseteq I_{n}(c)$. A clustering $C$ covers a set $S \subseteq X_{n}$ iff $S \subseteq \bigcup_{c \in C} I_{n}(c)$. The clustering $C$ is a prime clustering of a set $S$ iff $C$ covers $S$ and there is not a proper subset $C^{\prime} \subset C$ such that $C^{\prime}$ covers $S$. A prime clustering is therefore one that contains no extraneous clusters.

The goal of clustering is to find, given a finite subset $S \subseteq X_{n}$ of elements, a (prime) clustering that covers $S$ (and possibly other elements of $X_{n}$ ) such that each cluster of the clustering covers similar elements (i.e., is tight), and different clusters cover dissimilar elements (i.e., have large distance). The definitions of tightness and distance should depend only on clusters, i.e., on statements in the cluster description language, and not on the points covered by those clusters, thus fulfilling the primary condition of conceptual clustering (Michalski \& Stepp, 1983; Fisher \& Langley, 1985). 
The tightness function will be parameterized by $n$ in a manner similar to domains and clustering description languages. Since the clustering algorithm must employ this function, it is unreasonable to expect it to work for all $n$ unless the tightness functions for each $n$ are related to the extent that there is a single algorithm for evaluating them. For the same reason, the distance functions should be interrelated as well.

Definition 4 A family $\mathcal{F}=\left\{F_{n}\right\}_{n \geq 1}$ of functions is uniformly computable iff there is an algorithm $F$ such that for all $n$ and $x, F(n, x)=F_{n}(x)$. A family is uniformly polynomial-time computable iff $F$ runs in time polynomial in the value of $n$ and the size of $x$.

The formal definitions for tightness and distance functions are thus:

Definition $5 T=\left\{T_{n}\right\}_{n \geq 1}$ is a uniformly computable family of tightness functions, with $T_{n}: K_{n} \rightarrow \Re^{+}$, where $\Re^{+}$denotes the nonnegative real numbers.

Definition $6 D=\left\{D_{n}\right\}_{n \geq 1}$ is a uniformly computable family of distance functions, with $D_{n}: K_{n} \rightarrow \Re^{+}$.

$T_{n}$ is a measure of tightness of clusterings over the language $L_{n}$. Note that $T_{n}$ is a function of clusterings, and not of individual clusters. For monomial clustering, a natural measure of the tightness of a monomial is simply the number of attributes appearing in the monomial. A natural measure of overall tightness of a clustering (set of monomials) might be the minimum tightness of any monomial of the set. We define this tightness function $\tau$ for monomials in the next section.

$D_{n}$ is a measure of distance of clusterings over the language $L_{n}$. Note that $D_{n}$ is a function of clusterings, not of pairs of clusters. In the next section, we define the distance $D_{n}$ of a monomial clustering to be the minimum number of literals on which any pair of monomials of the clustering differ.

Numerical/statistical methods typically use a single metric that measures either similarity or dissimilarity between points. The objective function measures the overall quality of a clustering relative to that metric. For the sake of generality, we have assumed separate similarity (tightness) and dissimilarity (distance) measures, and allow the objective function (which we call "goodness") to be a function of these components.

Definition $7 \mathcal{G}=\left\{G_{n}\right\}_{n \geq 1}$ is a uniformly computable family of goodness functions, with $G_{n}: \operatorname{range}\left(T_{n}\right) \times \operatorname{range}\left(D_{n}\right) \rightarrow \Re^{+}$. 
The goodness $G_{n}$ of a clustering is a real number representing how well both tightness of clusters and distance between clusters has been achieved. We extend the domain of $G_{n}$ to $K_{n}$ with the natural interpretation that $\left(\forall C \in K_{n}\right) G_{n}(C)=G_{n}\left(T_{n}(C), D_{n}(C)\right)$. We are now ready to define clustering problems.

\section{Definition 8}

- $A$ (conceptual) clustering problem is any six-tuple $(\mathcal{X}, \mathcal{L}, I, \tau, \mathcal{D}, \mathcal{G})$ with $\mathcal{X}, \mathcal{L}, I, \mathcal{T}, D$, and $\mathcal{G}$ defined as above.

- An instance of a conceptual clustering problem $(\mathcal{X}, \mathcal{L}, I, \tau, \mathcal{D}, \mathcal{G})$ is a seven-tuple $\left(X_{n}, L_{n}, I_{n}, T_{n}, D_{n}, G_{n}, S\right)$, where $X_{n} \in \mathcal{X}, L_{n} \in \mathcal{L}$, $I_{n} \in I, T_{n} \in \mathcal{T}, D_{n} \in \mathcal{D}, G_{n} \in \mathcal{G}$, and $S$ is any finite nonempty subset of $X_{n}$.

- $A$ solution to an instance $\left(X_{n}, L_{n}, I_{n}, T_{n}, D_{n}, G_{n}, S\right)$ of a conceptual clustering problem is a clustering $C \in K_{n}$ (called a best clustering) such that

1. $C$ is a prime clustering of $S$.

2. For all clusterings $C^{\prime}$ that satisfy 1. above, $G_{n}\left(C^{\prime}\right) \leq G_{n}(C)$.

- An algorithm $A$ solves the conceptual clustering problem $(\mathcal{X}, \mathcal{L}, I$, $\tau, D, \mathcal{G})$ iff for all $n$ such that $X_{n}$ is nonempty, and for any finite nonempty set $S \subseteq X_{n}$, the algorithm $A$, if given $n$ and $S$ as input, outputs a solution to the instance $\left(X_{n}, L_{n}, I_{n}, T_{n}, D_{n}, G_{n}, S\right)$. We also write that $(\mathcal{X}, \mathcal{L}, I, \tau, \mathcal{D}, \mathcal{G})$ is solvable.

In what follows, the scope of the variable $n$ will be all numbers such that $X_{n}$ is nonempty. Thus the statement "for all $n$ " is used to mean "for all $n$ such that $X_{n}$ is nonempty."

Note that if $X_{n}$ is infinite, there may not exist a solution to $\left(X_{n}, L_{n}, I_{n}\right.$, $T_{n}, D_{n}, G_{n}, S$ ) because there may be an infinite sequence of clusterings for which $G_{n}$ increases without bound. Also note that the solution (if it exists) of an instance of a clustering problem may not induce a partition of the points of $S$. There is no requirement that the clusters of the solution cover disjoint sets, though disjointness may be enforced by an appropriate choice of $G_{n}$. Further, the clusters may cover (possibly an infinite number of) points of $X_{n}-S$.

These definitions, and the results in the following sections, are easily applied to the case of cluster analysis: the concept description language is simply finite subsets of $X_{n}$, and the interpretations I are identity functions $I_{n}$. Thus a cluster is simply a finite set of points of $X_{n}$. 
Since we are interested in feasible computations, we now define polynomial-time solvability of clustering problems. Recall that the parameter $n$ typically reflects a natural measure of size or length of encoding of objects $x \in X_{n}$.

Definition 9 Let the families $\tau, D$, and $\mathcal{G}$ be uniformly polynomial-time computable. Then $(\mathcal{X}, \mathcal{L}, I, \tau, D, \mathcal{G})$ is solvable in polynomial time iff there is an algorithm $A$ and polynomial $p$ such that

1. A solves the clustering problem $(\mathcal{X}, \mathcal{L}, I, \tau, \mathcal{D}, \mathcal{G})$;

2. For any $n$ (for which $X_{n}$ is nonempty), and any finite $S \subseteq X_{n}$, the run-time of $A$ on input $n$ and $S$ is at most $p(n,|S|)$.

\section{A restricted class of clustering problems}

The definitions in Section 2 are so general that it would be ridiculous to expect that all clustering problems are polynomially solvable (or even solvable, for that matter). In our framework, a main goal is to identify exactly those clustering problems that are (polynomially) solvable, and to give algorithms for solving them. Only by restricting the class of domains $\mathcal{X}$, the languages $\mathcal{L}$, and the objective functions $T, D$, and $\mathcal{G}$ under consideration, can we begin to make progress toward this goal. (As it turns out, we will not need to make any restrictions whatsoever on the domains $\chi$.)

A natural algorithm for clustering is the following: Given a set of elements $S$ to be clustered, begin by forming a cluster for each element of $S$. Then, iteratively "merge" the two clusters that are "closest." Halt when there is only one cluster remaining (containing all of the points of $S$ ) and output the best clustering encountered during this process. This is essentially the agglomerative algorithm formally specified in Section 4 .

In Section 3.1 we illustrate the agglomerative algorithm using an instance of the monomial clustering problem. In Section 3.2 we use the example to motivate properties for $\mathcal{L}, \tau . D$, and $\mathcal{g}$ that guarantee that the agglomerative algorithm finds an optimal solution.

\subsection{An example using the agglomerative algorithm}

The monomial clustering problem, discussed informally in the last section, is defined by:

- $\chi=\left\{X_{n}\right\}_{n \geq 1}$, where $X_{n}$ is the set of vectors over the variable set $x_{1}, x_{2}, \ldots, x_{n}$.

- $\mathcal{L}=\left\{L_{n}\right\}_{n \geq 1}$, where $L_{n}$ is the set of monomials over the same variables. ${ }^{4}$

\footnotetext{
${ }^{4}$ This is an example of the single representation trick (Cohen \& Feigenbaum, 1983).
} 
- $I=\left\{I_{n}\right\}_{n \geq 1}$, where $I_{n}$ is the standard logical interpretation, i.e., the interpretation of a monomial is the set of Boolean vectors that satisfy it.

- $T=\left\{T_{n}\right\}_{n \geq 1}$, where, if $C=\left\{m_{1}, m_{2}, \ldots, m_{k}\right\}$ is a clustering of $L_{n}$,

$$
T_{n}(C)=\min _{i=1, \ldots, k}\left\{t_{n}\left(m_{i}\right)\right\}
$$

and $t_{n}$, the tightness of a monomial, is the number of attributes (literals) in the monomial.

- $D=\left\{D_{n}\right\}_{n \geq 1}$, where, if $C=\left\{m_{1}, m_{2}, \ldots, m_{k}\right\}$ is a clustering of $L_{n}$,

$$
D_{n}(C)=\min _{1 \leq i \neq j \leq k}\left\{d_{n}\left(m_{i}, m_{j}\right)\right\},
$$

and $d_{n}$, the distance between two monomials, is the number of attributes that appear negated in one monomial and not negated in the other. If the clustering $C$ has only one monomial, arbitrarily define $D_{n}(C)=0$.

- $\mathcal{G}=\left\{G_{n}\right\}_{n \geq 1}$, where, if $C \in K_{n}$, then $G_{n}(C)=\min \left\{D_{n}(C), T_{n}(C)\right\}$.

The above objective functions $\tau, D$, and $\mathcal{G}$ capture the following three goals: (a) a tight clustering should contain only monomials that cover few points (a difference of 1 in the value of $t_{n}$ corresponds to a factor of 2 in the number of points of $X_{n}$ covered); (b) all monomials found should be disjoint (a clustering $C$ containing non-disjoint monomials $m_{1}$ and $m_{2}$ will have $D_{n}(C)=0$, since $d_{n}\left(m_{1}, m_{2}\right)=0$ ) and should differ on as many attributes as possible; (c) a small value of $T_{n}$ or $D_{n}$ is equally undesirable, since the overall goal is to maximize the minimum of the two measures.

To see how the agglomerative algorithm works on the monomial clustering problem, we give a sample run using the instance $\left(X_{9}, L_{9}, I_{9}, T_{9}, D_{9}\right.$, $G_{9}, S$ ), where the input set $S$ consist of the points (events) $e_{1}, \ldots, e_{5}$ as follows:

$$
\begin{aligned}
& e_{1}=x_{1} \bar{x}_{2} x_{3} x_{4} x_{5} x_{6} x_{7} \bar{x}_{8} \bar{x}_{9} \\
& e_{2}=\bar{x}_{1} x_{2} \bar{x}_{3} \bar{x}_{4} \bar{x}_{5} x_{6} \bar{x}_{7} x_{8} x_{9} \\
& e_{3}=x_{1} \bar{x}_{2} x_{3} x_{4} \bar{x}_{5} \bar{x}_{6} x_{7} \bar{x}_{8} \bar{x}_{9} \\
& e_{4}=\bar{x}_{1} x_{2} \bar{x}_{3} \bar{x}_{4} \bar{x}_{5} \bar{x}_{6} x_{7} x_{8} x_{9} \\
& e_{5}=x_{1} \bar{x}_{2} x_{3} x_{4} x_{5} \bar{x}_{6} \bar{x}_{7} \bar{x}_{8} \bar{x}_{9}
\end{aligned}
$$

For the rest of this section, we will refer to $X_{9}, L_{9}, I_{9}, T_{9}, D_{9}, G_{9}, t_{9}$, and $d_{9}$ as $X, L, I, T, D, G, t$, and $d$, respectively. 
Step 1. The agglomerative algorithm begins with the clustering

$$
C_{1}=\left\{m_{i}=e_{i}: 1 \leq i \leq 5\right\} .
$$

Note that this clustering is as "specific" as possible, in that each cluster is contained in some cluster of every monomial clustering that also covers $S$. The goodness of this clustering is 2, since all monomials have $t\left(m_{i}\right)=9$, and the minimum distance (between the pairs $\left(m_{1}, m_{3}\right),\left(m_{1}, m_{5}\right),\left(m_{2}, m_{4}\right)$, and $\left.\left(m_{3}, m_{5}\right)\right)$ is 2 .

Step 2. The agglomerative algorithm chooses one of the minimally-distant pairs for merging. Assume it picks the pair $\left(m_{1}, m_{5}\right)$. The obvious way to merge two monomials is to simply drop the attributes on which they differ. This results in a new clustering $C_{2}=\left\{m_{1}, \ldots, m_{4}\right\}$ where:

$$
\begin{aligned}
m_{1} & =x_{1} \bar{x}_{2} x_{3} x_{4} x_{5} \bar{x}_{8} \bar{x}_{9} \\
m_{2}=e_{2} & =\bar{x}_{1} x_{2} \bar{x}_{3} \bar{x}_{4} \bar{x}_{5} x_{6} \bar{x}_{7} x_{8} x_{9} \\
m_{3}=e_{3} & =x_{1} \bar{x}_{2} x_{3} x_{4} \bar{x}_{5} \bar{x}_{6} x_{7} \bar{x}_{8} \bar{x}_{9} \\
m_{4}=e_{4} & =\bar{x}_{1} x_{2} \bar{x}_{3} \bar{x}_{4} \bar{x}_{5} \bar{x}_{6} x_{7} x_{8} x_{9}
\end{aligned}
$$

The new cluster $m_{1}$ covers the events $e_{1}$ and $e_{5}$, as well as other points of $X_{n}$. Its tightness $t$ is 7 and the new minimum distance (between $m_{1}$ and $m_{3}$ ) is 1 . Therefore, the goodness of the new clustering is also 1 . Note that this is less than the goodness of the initial clustering; the algorithm does not hill-climb on this objective function.

Step 3. The algorithm merges the monomials $m_{1}$ and $m_{3}$. This results in a new clustering $C_{3}=\left\{m_{1}, m_{2}, m_{3}\right\}$ where:

$$
\begin{gathered}
m_{1}=x_{1} \bar{x}_{2} x_{3} x_{4} \bar{x}_{8} \bar{x}_{9} \\
m_{2}=e_{2}=\bar{x}_{1} x_{2} \bar{x}_{3} \bar{x}_{4} \bar{x}_{5} x_{6} \bar{x}_{7} x_{8} x_{9} \\
m_{3}=e_{4}=\bar{x}_{1} x_{2} \bar{x}_{3} \bar{x}_{4} \bar{x}_{5} \bar{x}_{6} x_{7} x_{8} x_{9}
\end{gathered}
$$

Step 4. The minimum tightness is $6\left(m_{1}\right)$ and the minimum distance is 2 (between $m_{2}$ and $m_{3}$ ). The algorithm therefore merges $m_{2}$ and $m_{3}$, resulting in $C_{4}=\left\{m_{1}, m_{2}\right\}$ where:

$$
\begin{gathered}
m_{1}=x_{1} \bar{x}_{2} x_{3} x_{4} \bar{x}_{8} \bar{x}_{9} \\
m_{2}=\bar{x}_{1} x_{2} \bar{x}_{3} \bar{x}_{4} \bar{x}_{5} x_{8} x_{9}
\end{gathered}
$$

This clustering has $T=6$ and $D=6$, so $G=6$. In fact, this is a best clustering of the events under the given objective function. 
Step 5. The last step in the example merges the two remaining clusters into a single monomial to obtain the clustering $C_{5}=\{\emptyset\}$ (the empty monomial) with $G\left(C_{5}\right)=T\left(C_{5}\right)=D\left(C_{5}\right)=0$. The algorithm therefore keeps $C_{4}$ as the best clustering.

\subsection{Properties of clustering problems}

Now we will present some properties for clustering problems, using the example from the previous subsection to provide intuitive motivations. For a clustering problem $(\mathcal{X}, \mathcal{L}, I, \tau, D, \mathcal{G})$, we state only the (more restrictive) properties sufficient for polynomial-time solvability; corresponding properties for general solvability are trivially obtained by dropping the polynomial-time requirements. In Section 4, we will prove that a clustering problem possessing the properties is solvable in polynomial time because the agglomerative algorithm meets the requirements of Definition 9.

In our example, the cluster description language and interpretation make it easy to determine whether a point $x \in X_{n}$ is covered by a statement $c \in L_{n}$. The ability to determine cluster membership is necessary if the agglomerative algorithm is to create prime clusterings - the merging operation used in the agglomerative algorithm may produce a new cluster whose interpretation is a superset of (the interpretation of) some cluster not involved in the merge. To guarantee prime clusterings, we must be able to detect such extraneous clusters. The first property therefore requires that we be able to determine cluster membership in polynomial time.

Property $P_{1}$. There exists a polynomial-time algorithm that, when given as input any number $n$, any point $x \in X_{n}$, and any cluster $c \in L_{n}$, outputs "true" if $x \in I_{n}(c)$ and "false" otherwise.

The membership algorithm may be used to obtain a prime clustering from a given clustering of a set $S$. In particular, let the polynomial-time subroutine PRIME $(n, C, S)$ return a prime clustering $C^{\prime}$ of $S$, where $C^{\prime}$ contains a subset of the clusters of $C$. PRIME starts with $C^{\prime}=C$, and iteratively removes clusters from $C^{\prime}$ until a prime clustering of $S$ is obtained. (A cluster $c$ is removed from $C^{\prime}$ iff every point in $S$ that is covered by $c$ is also covered by some other cluster of $C^{\prime}$.)

The next property is based on step 1 of the example. In this step, the algorithm created a single cluster for each point $x_{i} \in S$. Each cluster $c_{i}$ covered $x_{i}$ and as few additional points of $X$ as possible. For some languages $L_{n}$ and some sets $S$, there may not exist a clustering of individual points that is "most specific" in this sense. Property $P_{2}$ asserts that the cluster description languages must be such that for any set of points in $X_{n}$, there must exist a cluster (statement in $L_{n}$ ) that is the most specific 
of any statement in $L_{n}$ covering those points, and there must exist feasible (polynomial) means for finding this cluster.

Definition 10 For a given clustering problem $(\mathcal{X}, \mathcal{L}, I, \mathcal{T}, \mathcal{D}, \mathcal{G})$, and for any $n$, a maximally specific cover (MSC) for a set of points $P \subseteq X_{n}$ is a cluster $c \in L_{n}$ such that $c$ covers $P$, and for any $c^{\prime} \in L_{n}$, if $c^{\prime}$ covers $P$, then $I_{n}(c) \subseteq I_{n}\left(c^{\prime}\right)$.

Property $P_{2}$. For any number $n$ and finite $S \subseteq X_{n}$, there exists a unique MSC for $S$. Further, there exists an algorithm that, when given as input $n$ and $S=\left\{x_{1}, x_{2}, \ldots x_{s}\right\}$, outputs a clustering $C=\left\{c_{1}, c_{2}, \ldots, c_{s}\right\} \in K_{n}$ such that for $1 \leq i \leq s, c_{i}$ is the MSC for $\left\{x_{i}\right\}$. The run-time of the algorithm must be polynomial in $n$ and $|S|$.

Property $P_{2}$ implies that the MSCs (descriptions) of the singleton point sets $\left\{c_{i}\right\}$ be at most of size polynomial in $n$ and $|S|$.

The next two properties are based on the merging operation in the example. The agglomerative algorithm assumes that $D(C)$ for a clustering is based on an inter-cluster distance measure $d$.

Property $P_{3}$. There is a family of uniformly polynomial-time computable functions $d=\left\{d_{n}\right\}$, where $d_{n}: L_{n} \times L_{n} \rightarrow \Re^{+}$, such that for all $n$, and $C \in K_{n}$ :

(a) $D_{n}(C)=\min \left\{d_{n}\left(c_{i}, c_{j}\right): c_{i}, c_{j} \in C, c_{i} \neq c_{j}\right\}$. When restricted to single-cluster clusterings, $\left\{D_{n}\right\}$ may be any family of uniformly polynomial-time computable functions satisfying (b) below.

(b) $\left(\forall c_{1}, c_{2}, c_{3}, c_{4} \in L_{n}\right) \quad\left(I_{n}\left(c_{1}\right) \subseteq I_{n}\left(c_{2}\right)\right)$ and $\left(I_{n}\left(c_{3}\right) \subseteq I_{n}\left(c_{4}\right)\right) \Rightarrow$ $d_{n}\left(c_{1}, c_{3}\right) \geq d_{n}\left(c_{2}, c_{4}\right)$. If $C_{1}=\left\{c_{1}\right\}$ and $C_{2}=\left\{c_{2}\right\}$ are clusterings containing only single clusters, then $I_{n}\left(c_{1}\right) \subseteq I_{n}\left(c_{2}\right) \Rightarrow D_{n}\left(C_{1}\right) \geq D_{n}\left(C_{2}\right)$.

Property $P_{3}$ requires that the distance $D$ of a clustering really is the minimum inter-cluster "distance" $d$ between any pair of clusters, where $d$ has a monotone property: If two clusters have distance $d$ and points are then added to each, the distance $d$ cannot increase. In other words, as clusters "grow," the distance between them shrinks, and $D$ is the minimum of all these inter-cluster distances.

It is not clear what is meant by the "distance" of a clustering $C$ when $C$ contains only a single cluster. Generally, one is only interested in clusterings that contain more than one cluster. A possible way to deal with this is to simply let the value of $G$ be zero for any such clustering, or to let the value of $D$ be zero. For the sake of generality, we have chosen a weaker requirement, which is to allow $D$ to be defined arbitrarily for one-cluster clusterings, but to require that $D$ be monotone under generalization. Perhaps more natural, but also more restrictive, would be to require that the 
value of $D$ be the same for all one-cluster clusterings, or to let $G$ be defined as a function of $T(C)$ alone when $C$ has only one cluster.

In steps 2-4 of the example, the algorithm merged monomials in an obvious way to produce new clusters. Also, the clusters produced by merging monomials were always maximally specific covers (for the points covered by the merged clusters). Property $P_{4}$ is related to $P_{2}$. It requires that we be able to generate, in polynomial time, an MSC for the union of any two sets of points in the problem space described by clusters:

Property $P_{4}$. There is an effective procedure $M$ such that for any $n, M$ "merges" any two clusters $c, c^{\prime} \in L_{n}$. For all $n, M$ and $L_{n}$ must have the following properties:

(a) For all $c, c^{\prime} \in L_{n}$, there is a unique MSC $c^{\prime \prime}$ for $I_{n}(c) \cup I_{n}\left(c^{\prime}\right)$, and $M\left(n, c, c^{\prime}\right)=c^{\prime \prime}$.

(b) $M$ runs in time polynomial in $n$ and in the lengths of the statements $c$ and $c^{\prime}$.

(c) There is a polynomial $q$ such that for any finite subset $S$ of $X_{n}$, if $c$ is obtained by any (finite) number of merges of MSCs of subsets of $S$, then $c$ has size at most $q(n,|S|)$.

Since the agglomerative algorithm successively "merges" clusters, it is possible that at some point, the description of a cluster is larger than any given polynomial in the size of $S$. Part (c) assures that this event will not occur. Note that no reasonable restriction on the size of the statement $M\left(n, c, c^{\prime}\right)$ in part (b) is sufficient to prevent this event. For example, since there are at most $|S|$ iterations, even if we require that the length of the statement $M\left(n, c, c^{\prime}\right)$ is at most the sum of the lengths of the statements $c$ and $c^{\prime}$, it is possible that the final clustering obtained by the algorithm will have size exponential in $|S|$. By requiring part (c) in addition to part (b), we guarantee that any statement produced by the algorithm has size at most polynomial in the size of $S$.

In many cases (e.g., when clusters are conjunctive descriptions over any collection of attributes), the size of descriptions will decrease as clusters become more general. In other cases (e.g., axis-aligned rectangles in Euclidean spaces), description size will remain constant. In cluster analysis, property $P_{4}$ is trivially satisfied, since merging is done by union (of sets of points), and the largest statement is exactly $S$.

Property $P_{5}$ is based on the observation that, in the monomial example, clusters became less cohesive (more general or less tight) as merges occurred. We need the following definition:

Definition 11 Given $X_{n}, L_{n}$, and $I_{n}$, the relation $\unlhd_{n}$ on $K_{n}$ is defined by: For all $C, C^{\prime} \in K_{n}, C \unlhd_{n} C^{\prime}$ iff $(\forall c \in C)\left(\exists c^{\prime} \in C^{\prime}\right) I_{n}(c) \subseteq I_{n}\left(c^{\prime}\right)$. 
If $C \unlhd_{n} C^{\prime}$ we say $C$ is less general than, more specific than, and is a specialization of, $C^{\prime}$, and equivalently, that $C^{\prime}$ is more general than, less specific than, and is a generalization of, $C$.

When restricted to prime clusterings with respect to a given set $S, \unlhd_{n}$ is a partial order on $K_{n}$.

Property $P_{5}$. The tightness functions $T=\left\{T_{n}\right\}$ are a uniformly polynomial-time computable family of functions, and for all $n$, the function $T_{n}$ is monotone nonincreasing under generalization, i.e., for all $C, C^{\prime} \in K_{n}$, if $C \unlhd_{n} C^{\prime}$ then $T_{n}(C) \geq T_{n}\left(C^{\prime}\right)$.

Property $P_{5}$ asserts that if one clustering is a generalization of another, then the more general clustering is at most as tight as the less general one. Because we have defined tightness as a function of clusterings and not of individual clusters, it is not immediately clear that this is a natural property. However, observe that the definition of the relation $\unlhd_{n}$ states that each cluster of the less general clustering is contained in some cluster of the more general clustering. Thus if $T_{n}$ somehow depends on the "tightness" of particular clusters (e.g., if tightness of individual clusters is inversely related to the quantity or variety of elements covered), then the more general clustering contains clusters at most as tight as the clusters of the less general clustering. We would then expect that the overall value of $T_{n}$ would be greater for the less general clustering.

Finally, property $P_{6}$ simply states that goodness has a very natural property: If one increases either distance or tightness while holding the other constant, then goodness should not decrease. In other words, tight, distant clusterings are best.

Property $P_{6}$. The goodness functions $\mathcal{G}=\left\{G_{n}\right\}$ are a uniformly polynomial-time computable family of functions, and for all $n$, the function $G_{n}$ is monotone nondecreasing in $T_{n}$ and $D_{n}$. That is, if $x_{1} \geq x_{2} \in \operatorname{range}\left(T_{n}\right)$ and $y_{1} \geq y_{2} \in \operatorname{range}\left(D_{n}\right)$, then $G_{n}\left(x_{1}, y_{1}\right) \geq G_{n}\left(x_{2}, y_{1}\right)$ and $G_{n}\left(x_{1}, y_{1}\right) \geq$ $G_{n}\left(x_{1}, y_{2}\right)$.

\subsection{Example clustering problems}

The properties $P_{1}$ through $P_{6}$ hold for several interesting conceptual clustering and cluster analysis problems that fall within our framework. In this section, we present some of these, without proof that they do indeed satisfy the properties.

Monomial clustering. The properties $P_{1}-P_{6}$ were motivated by, and are natural generalizations of, properties held by the monomial clustering problem. It is easily verified that these properties are satisfied by the monomial clustering problem as defined in Section 3.1. The properties also 
hold for conjunctive conceptual clustering using multiple-valued attributes and internal disjunction (Michalski \& Stepp, 1983). ${ }^{5}$ In this case, the initial clustering is the same as for monomials and the "refunion" operator (Michalski, 1983) can be used for merging. Natural extensions of the distance and tightness measures in the example satisfy $P_{3}$ and $P_{5}$, and these may be used with any objective function satisfying $P_{6}$.

Geometric clustering. Another interesting group of languages that have these properties are some geometric languages over Euclidean spaces. For example, the agglomerative algorithm can solve the axis-aligned rectangle clustering problem, defined by:

- $\chi=\left\{X_{n}\right\}$, where $X_{n}$ is $n$-dimensional Euclidean space.

- $\mathcal{L}=\left\{L_{n}\right\}$, where $L_{n}$ is the set of $n$-dimensional rectangles with sides parallel to the $n$ axes.

- $I=\left\{I_{n}\right\}$ is the standard interpretation: $I_{n}(r)$, is the set of points of $X_{n}$ that are contained in $r$, where $r$ is a rectangle of $L_{n}$.

- $\tau=\left\{T_{n}\right\}$, where $T_{n}(\mathrm{C})$ for a collection of rectangles $C$ could be any of:

1. The inverse of the area of the union of the rectangles of $C$.

2. The inverse of the area of the largest rectangle of $C$.

3. The inverse of the maximum distance between any two points within any cluster, i.e., the inverse of the length of the longest diagonal.

- $\mathrm{D}=\left\{D_{n}\right\}$, where $D_{n}(\mathrm{C})$ is the minimum pairwise "distance" $d_{n}$ between any pair of rectangles of $C$, and $d_{n}$ is any metric that gets smaller as clusters grow. ${ }^{6}$ Let $D_{n}(C)=0$ if $C$ has only one cluster.

- $\mathcal{G}=\left\{G_{n}\right\}$ is any objective function satisfying $P_{6}$.

The critical condition for geometric languages is that there exist, and one can find, a description of the smallest set representable in the language that covers a given set of points. For example, if the language consists of descriptions of all convex polygons in 2-dimensional Euclidean space, then it is easy to see that the agglomerative algorithm may be successfully applied, since the convex hull of a set of points (which may be found in polynomial time) is contained in every convex polygon containing the points. However, if $\mathcal{L}=\left\{L_{n}\right\}$, where $L_{n}$ consists of descriptions of convex polytopes in $n$ dimensions (i.e., a list of $(n-1)$-dimensional hyperplanes),

\footnotetext{
${ }^{5}$ Some of the metrics used by Michalski and Stepp, e.g., "simplicity" and "sparseness", clearly do not satisfy properties $P_{3}$ and $P_{5}$.

${ }^{6}$ Some metrics $d_{n}$ that $d o$ not have this property include $d_{n}\left(r_{1}, r_{2}\right)=$ maximum distance between any pair of points of $r_{1}$ and $r_{2}$, or the distance between the centers of $r_{1}$ and $r_{2}$.
} 
then property $P_{2}$ (and $P_{4}$ ) do not hold, because the length of a description of the convex hull of a set of $s$ points in $n$ dimensions (i.e., the length of the description of the MSC of a set of points) can be as large as $s^{\left\lfloor\frac{n}{2}\right\rfloor}$ (Edelsbrunner, 1987). If one is willing to relax the requirement that the clustering found have size polynomial in the dimension, then the agglomerative algorithm can be used to find an optimal clustering. The MSCs are obtained by applying any algorithm for finding the convex hull of a set of points in $n$ dimensions.

Cluster Analysis. Within our framework, any cluster analysis problem trivially satisfies properties $P_{1}, P_{2}$, and $P_{4}$. Whether properties $P_{3}, P_{5}$, and $P_{6}$ are satisfied will depend on the particular choice of the objective functions. Single linkage clustering (Anderberg, 1973), in which the distance between clusters ( $d_{n}$ in our framework) is the minimum distance between points in the clusters, clearly satisfies the monotone requirement for distance metrics in property $P_{3}$. Complete linkage clustering $\left(d_{n}\right.$ is the maximum inter-point distance) clearly does not. Most reasonable choices of $\mathcal{G}$ will satisfy property $P_{6}$.

\section{The agglomerative algorithm}

We now formally introduce the agglomerative algorithm and prove that it solves any clustering problem satisfying properties $P_{1}$ through $P_{6}$. In Section 4.2 we examine further characteristics of the algorithm, and in Section 4.3 we present results concerning variants of the tightness and distance measures.

\subsection{Algorithm $A$}

The algorithm we consider is the Central Agglomerative Procedure as described by Anderberg (1973). Variants of this method constitute the majority of the work on hierarchical clustering (Romesburg, 1984). Hierarchical clustering techniques are normally used to produce a classification tree over the object set, where leaves are individual objects and internal nodes represent clusters. We will instead be concerned with whether the technique finds a single clustering that is best under the objective function. In this sense, we are using the agglomerative/hierarchical procedure as an optimization technique (Everitt, 1980), but without fixing the number of clusters beforehand. We are also allowing the algorithm to produce non-disjoint clusters, as in clumping techniques (Everitt, 1980).

Table 1 specifies the basic agglomerative algorithm $\mathrm{A}$. Given $n$ and a finite nonempty set $S \subseteq X_{n}$, the algorithm produces $t \leq|S|$ different clusterings $C_{1}, C_{2}, \ldots, C_{t}$, by starting with the maximally specific cluster for 
Table 1. Agglomerative algorithm $A$.

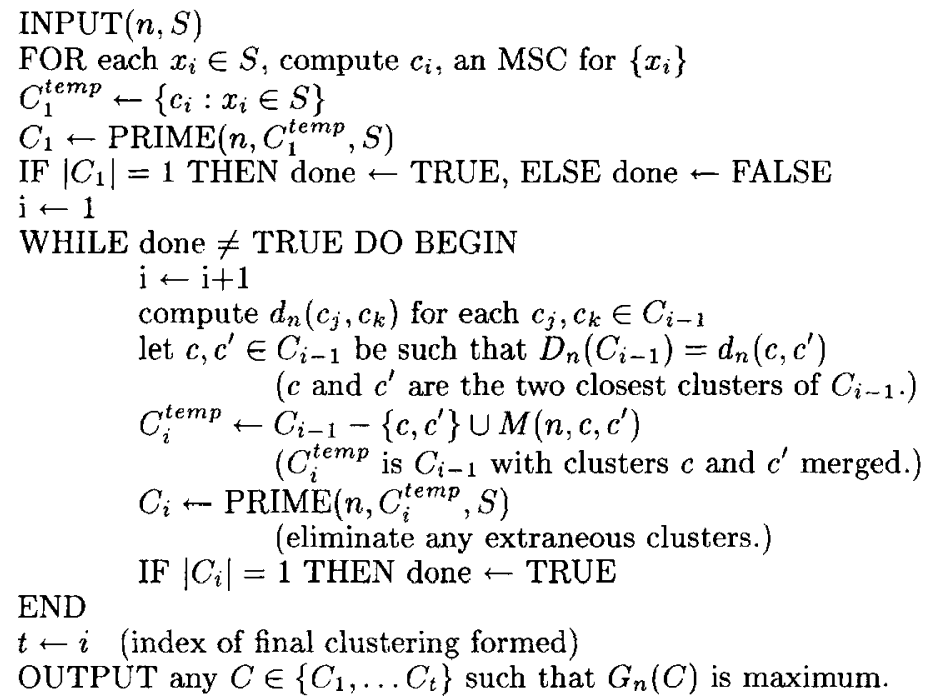

each point in $S$ and successively merging clusters with minimum distance until a single cluster covering all of $S$ is obtained. After each merge, extraneous clusters are eliminated. The output of the algorithm is the clustering among $C_{1}, C_{2}, \ldots C_{t}$ with the best value. We will prove that the algorithm solves any clustering problem $(\mathcal{X}, \mathcal{L}, I, \tau, \mathcal{D}, \mathcal{G})$ that satisfies properties $P_{1}$ through $P_{6}$. (The algorithm itself implicitly assumes that properties $P_{1}, P_{2}, P_{3}$ (a), and $P_{4}$ hold.)

Theorem 1 If $(\mathcal{X}, \mathcal{L}, I, \tau, D, \mathcal{G})$ is any clustering problem for which properties $P_{1}$ through $P_{6}$ are satisfied, then algorithm $A$ solves $(\mathcal{X}, \mathcal{L}, I$, $\tau, D, \mathcal{G})$ in polynomial time.

ProOF: By property $P_{2}$, the clustering $C_{1}^{\text {temp }}$ is found in polynomial time. Note that $d=\left\{d_{n}\right\}, D=\left\{D_{n}\right\}, \tau=\left\{T_{n}\right\}$, and $\mathcal{G}=\left\{G_{n}\right\}$ are uniformly polynomial-time computable families (properties $P_{3}, P_{5}$, and $P_{6}$ ), and that the merge operation $M$ never produces a statement of length greater than $q(n,|S|)$ (property $P_{4}$ (b) and (c)). Subroutine PRIME runs in polynomial time by the comments following the introduction of property $P_{1}$. Since there are $t \leq|S|$ iterations (because $\left|C_{i+1}\right|<\left|C_{i}\right|$ ), the algorithm runs in time polynomial in $|S|$ and $n$. We need only show that the algorithm is correct. 
Lemma 1 Let $\left(X_{n}, L_{n}, I_{n}, T_{n}, D_{n}, G_{n}, S\right)$ be an instance of a clustering problem that satisfies properties $P_{1}$ through $P_{6}$. Let $C \in K_{n}$ be a prime clustering of $S$, a specialization of some best clustering, and suppose that $C$ itself is not a best clustering. Let $c, c^{\prime} \in C$ be such that $D_{n}(C)=d_{n}\left(c, c^{\prime}\right)$, and let $C^{\prime}=C-\left\{c, c^{\prime}\right\} \cup M\left(n, c, c^{\prime}\right)$. (In other words, $C^{\prime}$ is obtained from $C$ by merging two clusters with minimum distance $d_{n}$.) Then $C^{\prime}$ is a specialization of a best clustering, as is $\operatorname{PRIME}\left(n, C^{\prime}, S\right)$.

We first show that Theorem 1 follows from Lemma 1, and then prove Lemma 1 . To prove the theorem, we need only show that at least one of the clusterings $\left\{C_{1}, C_{2}, \ldots, C_{t}\right\}$ is a best clustering.

Suppose by way of contradiction that none of the $C_{i}$ 's is a best clustering. By the definition of the maximally specific cover (MSC) of a set of points $P$, any cluster that covers $P$ must cover a superset of the points covered by the MSC. $C_{1}^{t e m p}$ consists of the MSCs for each point in $S$. Thus, $C_{1}^{t e m p}$ is a specialization of every clustering of $S$ and therefore of a best clustering. Trivially, $C_{1}$ is a specialization of a best clustering and is a prime clustering of $S$. By Lemma 1 and its definition, $C_{2}^{t e m p}$ is a specialization of a best clustering, as is $C_{2}$. Iteratively applying Lemma 1 and our supposition that none of the $C_{i}$ 's are best, we have that each of $C_{1}, C_{2}, \ldots, C_{t}$ is a specialization of a best clustering. (Each is also a prime clustering of $S$.) But this is a contradiction, for $C_{t}$ cannot be a specialization of a best clustering without being a best clustering: since $C_{t}$ has only one cluster, and only prime clusterings are candidate solutions, any generalization $C_{b e s t}$ of $C_{t}$ must have exactly one cluster that contains the single cluster of $C_{t}$. Further, $T_{n}\left(C_{\text {best }}\right) \leq T_{n}\left(C_{t}\right)$ and $D_{n}\left(C_{b e s t}\right) \leq D_{n}\left(C_{t}\right)$ by properties $P_{5}$ and $P_{3}$ (b). By $P_{6}, G_{n}\left(C_{b e s t}\right) \leq G_{n}\left(C_{t}\right)$, and thus $C_{t}$ is in fact a best clustering. It follows that our supposition was wrong, and at least one of $\left\{C_{1}, C_{2}, \ldots, C_{t}\right\}$ must be a best clustering.

We now prove Lemma 1. Let $C_{b e s t}$ be a best clustering, with $C \unlhd_{n} C_{b e s t}$, and $C$ a prime clustering of $S$, but not a best clustering. Then $G_{n}(C)<$ $G_{n}\left(C_{b e s t}\right)$.

Let $c, c^{\prime}$, and $C^{\prime}$ be as defined in the lemma. (Thus $d_{n}\left(c, c^{\prime}\right)=D_{n}(C)$.) Since $C \unlhd_{n} C_{b e s t}$, there are clusters $b, b^{\prime} \in C_{b e s t}$ such that $I_{n}(c) \subseteq I_{n}(b)$ and $I_{n}\left(c^{\prime}\right) \subseteq I_{n}\left(b^{\prime}\right)$. There are now two cases:

CASE 1: $b=b^{\prime}$. In this case, the only cluster of $C^{\prime}$ that is not also a cluster of $C$ is $M\left(n, c, c^{\prime}\right)$. Observe that $I_{n}(c) \cup I_{n}\left(c^{\prime}\right) \subseteq I_{n}(b)$, and by the definition of $M\left(n, c, c^{\prime}\right)$ as maximally specific, $I_{n}\left(M\left(n, c, c^{\prime}\right)\right) \subseteq I_{n}(b)$. Thus $C^{\prime}$ is a specialization of $C_{b e s t}$. Trivially, $\operatorname{PRIME}\left(n, C^{\prime}, S\right)$ is also a specialization of $C_{b e s t}$, and the lemma is proved. 
CASE 2: $b \neq b^{\prime}$. In this case, $T_{n}(C) \geq T_{n}\left(C_{b e s t}\right)$ by property $P_{5}$, and now note that:

$$
\begin{aligned}
& \left.D_{n}(C)=d_{n}\left(c, c^{\prime}\right) \quad \text { (by choice of } c, c^{\prime}\right) \\
& \geq d_{n}\left(b, b^{\prime}\right) \quad \text { (by property } P_{3}(\mathrm{~b}) \text { ) } \\
& \geq D_{n}\left(C_{b e s t}\right) \quad \text { (by property } P_{3}(\mathrm{a}) \text { ). }
\end{aligned}
$$

Since $T_{n}(C) \geq T_{n}\left(C_{b e s t}\right)$ and $D_{n}(C) \geq D_{n}\left(C_{\text {best }}\right)$, by property $P_{6}, G_{n}(C) \geq$ $G_{n}\left(C_{\text {best }}\right)$, contradicting the hypothesis of the lemma that $C$ is not a best clustering. Thus case 1 must hold, completing the proof of Lemma 1 and Theorem 1.

\subsection{Properties of algorithm $A$}

It is interesting to note that algorithm $A$ is not a hill-climbing method, in that the value of the objective function may increase and decrease as the sequence of clusters $C_{1}, C_{2}, \ldots, C_{t}$ is formed. (Recall the monomial example in Section 3.1.) However, it is true that the measure of tightness $T_{n}$ is monotone nonincreasing as each new clustering is examined. The function $D_{n}$ is not necessarily monotone, because it is possible for $D_{n}$ to increase when two clusters are merged (since the minimum distance is eliminated), and also to decrease (since the new larger cluster may be very close to some other cluster). It is for this reason that the algorithm must continue generating clusterings rather than stopping once the value of $G_{n}$ decreases. Under some objective functions, the algorithm may hillclimb. For example, single-linkage cluster analysis problems (Anderberg, 1973) define $d_{n}$ as the minimum "distance" between points of two clusters (where "distance" is any metric). If such a problem satisfies properties $P_{1}-P_{6}$, then the agglomerative algorithm will hill-climb on the objective function.

It is also worth noting that, for each $k \leq s$, algorithm $A$ finds the best clustering with at least $k$ clusters. We will show that for $1 \leq k \leq s$, the best clustering with at least $k$ clusters is in the set $\left\{C_{1}, C_{2}, \ldots, C_{t}\right\}$. This is achieved by proving, for each fixed $k \leq s$, the following variant of Lemma 1 . Let best ${ }_{k}$ mean "best among all prime clusterings of $S$ with at least $k$ clusters."

Lemma 2 Let $\left(X_{n}, L_{n}, I_{n}, T_{n}, D_{n}, G_{n}, S\right)$ be an instance of a clustering problem that satisfies properties $P_{1}$ through $P_{6}$. Let $C \in K_{n}$ be a prime clustering of $S$ containing at least $k$ clusters. Further, let $C$ be a specialization of some best ${ }_{k}$ clustering, and suppose that $C$ itself is not a best $k_{k}$ clustering. Let $c, c^{\prime} \in C$ be such that $D_{n}(C)=d_{n}\left(c, c^{\prime}\right)$, and let $C^{\prime}=C-\left\{c, c^{\prime}\right\} \cup M\left(n, c, c^{\prime}\right)$. (In other words, $C^{\prime}$ is obtained from $C$ by merging two clusters with minimum distance $d_{n}$.) Then $C^{\prime}$ is a specialization of a best ${ }_{k}$ clustering, as is $\operatorname{PRIME}\left(n, C^{\prime}, S\right)$. 
Lemma 2 differs from Lemma 1 only in that "best clustering" has been replaced with "best $t_{k}$ clustering" and the additional hypothesis that $C$ has at least $k$ clusters has been added. The proof of Lemma 2 is nearly identical to the proof of Lemma 1. (One needs the fact that $C$ has at least $k$ clusters to arrive at the contradiction in Case 2.) We can now prove

Theorem 2 If $(\mathcal{X}, \mathcal{L}, I, \tau, \mathcal{D}, \mathcal{G})$ is any clustering problem for which properties $P_{1}$ through $P_{6}$ are satisfied, then for each $k \leq|S|$, the set of clusterings $\left\{C_{1}, C_{2}, \ldots, C_{t}\right\}$ produced by algorithm $A$ contains a best ${ }_{k}$ clustering (if one exists).

PROOF: To prove Theorem 2, we assume that for some $k \leq|S|$, a best $t_{k}$ clustering exists (one could fail to exist because every prime clustering could have fewer than $k$ clusters), and that none of the (prime) clusterings $\left\{C_{1}, C_{2}, \ldots, C_{t}\right\}$ is a best $k_{k}$ clustering. We then obtain a contradiction.

Consider the sequence of clusterings $C_{1}^{t e m p}, C_{1}, C_{2}^{t e m p}, C_{2}, \ldots, C_{t}^{t e m p}, C_{t}$, produced during the run of algorithm $A$. Since $C=C_{1}^{t e m p}$ is such that

(a) $C$ has at least $k$ clusters and

(b) $C$ is a specialization of a best ${ }_{k}$ clustering,

there is a rightmost element $R$ of this sequence of clusterings that satisfies (a) and (b). There are three cases, each resulting in a contradiction:

CASE 1: For some $i, 1 \leq i<t, R=C_{i}$. Since $R$ is a prime clustering of $S$, by (a), (b), our assumption that none of the $C_{i}$ 's is a best $t_{k}$ clustering, and Lemma 2, we conclude that $C_{i+1}^{t e m p}$ is a specialization of a best $t_{k}$ clustering. Then $C_{i+1}^{\text {temp }}$ must have less than $k$ clusters, otherwise $R=C_{i+1}^{t e m p}$ instead of $C_{i}$. Since $C_{i}$ and $C_{i+1}^{t e m p}$ differ in number of clusters by exactly one, it follows that $C_{i}$ has exactly $k$ clusters. Let $C_{b e s t}$ be a (prime) generalization of $C_{i}$ that is a best ${ }_{k}$ clustering. Then $C_{\text {best }}$ must have exactly $k$ clusters, each a superset of a different cluster of $C_{i}$. Thus $T_{n}\left(C_{i}\right) \geq T_{n}\left(C_{b e s t}\right), D_{n}\left(C_{i}\right) \geq$ $D_{n}\left(C_{\text {best }}\right)$, and $G_{n}\left(C_{i}\right) \geq G_{n}\left(C_{b e s t}\right)$, contradicting the assumption that $C_{i}$ is not a best $k$ clustering.

CASE 2: $R=C_{t}$. Since $C_{t}$ has exactly one cluster, $k=1$. In other words, $C_{t}$ has exactly $k$ clusters, and the reasoning concluding case 1 above may be employed.

CASE 3: For some $i, 1 \leq i \leq t, R=C_{i}^{t e m p}$. Since $C_{i}^{\text {temp }}$ is a specialization of some best $k_{k}$ clustering $C_{\text {best }}$, so is $C_{i}$. Then $C_{i}$ must have less than $k$ clusters, otherwise $R=C_{i}$. Both $C_{i}$ and $C_{b e s t}$ are prime clusterings of $S$, so $C_{b e s t}$ can have at most the same number of clusters as $C_{i}$ (one superset of each $c \in C_{i}$ ). Therefore, $C_{b e s t}$ has less than $k$ clusters, and is not a best $k_{k}$ clustering, a contradiction. 
Since in each case we have arrived at a contradiction, our assumption that none of the clusterings $\left\{C_{1}, C_{2}, \ldots, C_{t}\right\}$ is a best ${ }_{k}$ clustering must be false, completing the proof of Theorem 2 .

A natural question is whether it is possible to find a best clustering with exactly $k$ clusters. Certainly this is at least as difficult as finding a best clustering with at most $k$ clusters, since an algorithm for the former problem could be run $k$ times to find best clusterings with exactly $1,2,3, \ldots, k$ clusters, and the best could be chosen as an answer to the latter problem. We show that there exists a clustering problem satisfying $P_{1}-P_{6}$ such that unless $P=N P$, no polynomial-time algorithm is guaranteed to find, for all instances of the problem, a best clustering with at most $k$ clusters.

It would appear that a simple reduction from the NP-hard CLUSTERING problem (Garey \& Johnson, 1979) would be sufficient to show this. However, due to the definition of an "instance" for each problem, a straightforward approach relating the "distance" function of CLUSTERING to any of our measures $\tau, D$, or $\mathcal{g}$ will not work.

We sketch a proof that there is a clustering problem $(\mathcal{X}, \mathcal{L}, I, \tau, D$, $\mathcal{G})$ satisfying $P_{1}$ through $P_{6}$ such that for each number $k \geq 3$, the problem of finding for all instances, $\left(X_{n}, L_{n}, I_{n}, T_{n}, D_{n}, G_{n}, S\right)$, a best clustering among those with at most $k$ clusters is NP-hard. Our example is a cluster analysis problem, thus for each $n, L_{n}=\left\{c: c\right.$ is a finite subset of $\left.X_{n}\right\}$, and $I_{n}$ is the identity function. As in all cluster analysis problems within our framework, properties $P_{1}, P_{2}$, and $P_{4}$ hold immediately.

Let $k \geq 3$ be given, and let $\chi, \tau, D$, and $\mathcal{G}$ be defined in the following manner. $X=\left\{X_{2 v}\right\}$, where $X_{2 v}$ is the set of (even) length $2 v$ strings. A given string of length $2 v$ will represent a vertex in an undirected graph of $v$ vertices if the first half of the string contains a single " 1 " bit. The single " 1 " among the first $v$ bits indicates which vertex it is, and the remaining $v$ bits give adjacency information with other vertices, i.e., a "1" in position $v+j$ indicates that the vertex is adjacent to vertex $j$. Note that any graph with $v$ vertices may be represented by a finite set of points of $X_{2 v}$, although not every finite subset of $X_{2 v}$ represents a graph. For example, the graph of 5 vertices with edges $(1,2),(1,3),(1,5),(2,3),(4,5),(3,5)$ corresponds to the subset of $X_{10}$ given by the elements $\left\{x_{1}, x_{2}, x_{3}, x_{4}, x_{5}\right\}$ in Table 2 . We have inserted a comma between the 5 th and 6 th bits and have parenthesized each element to aid the interpretation.

Given as input any even number $2 v$ and finite subset $S$ of $X_{2 v}$, it is decidable in polynomial time whether $S$ represents a subset of the vertices of some undirected graph of $v$ vertices, or whether no undirected graph has a subset of vertices represented by the elements of $S$. (What must be checked is that (1) each string of $S$ has a single "1" among the first $v$ bits; 
Table 2. A graph represented by bit strings.

$\begin{array}{ll}x_{1}=(10000,01101) & (\text { vertex } 1 \text { adjacent to } 2,3, \text { and } 5) \\ x_{2}=(01000,10100) & (\text { vertex } 2 \text { adjacent to } 1 \text { and } 3) \\ x_{3}=(00100,11001) & (\text { vertex } 3 \text { adjacent to } 1,2, \text { and } 5) \\ x_{4}=(00010,00001) & (\text { vertex } 4 \text { adjacent to } 5) \\ x_{5}=(00001,10110) & (\text { vertex } 5 \text { adjacent to } 1,3, \text { and } 4)\end{array}$

(2) for each $i \leq v$, there is at most one string in $S$ with a single " 1 " in position $i$; and (3) if a string representing a vertex numbered $i$ has a " 1 " in position $v+j$, then the string representing vertex $j$ (if it appears in $S$ ) has a " 1 " in position $v+i$.)

For a clustering $C$, let $T_{2 v}(C)=0$ if the union of the clusters of $C$ is not a finite subset of $X_{2 v}$ representing a subset of the vertices of some undirected graph, or if there is a cluster $c \in C$ such the representations of two vertices that are adjacent in the represented subgraph are both contained in $c$. Let $T_{2 v}(C)=1$ otherwise. In the example in Table 2, $T_{10}\left(\left\{\left\{x_{1}, x_{4}\right\},\left\{x_{2}, x_{5}\right\}\right\}\right)=1$, since the elements of the clusters are consistent with some 5 vertex undirected graph, and no two adjacent vertices appear in any single cluster. On the other hand, $T_{10}\left(\left\{\left\{x_{1}, x_{2}\right\},\left\{x_{3}\right\}\right\}\right)=0$, since in any graph that contains the vertices $x_{1}, x_{2}$, and $x_{3}, x_{1}$ is adjacent to $x_{2}$ and they appear in the same cluster. As a final example, $T_{4}(\{\{1001\},\{0100\}\})=0$, because the adjacency information between vertex 1 and 2 in the two vertex graph represented is inconsistent.

Let $D_{2 v}$ be the constant function $D_{2 v}(C)=2 v$, and let $G_{2 v}(C)=$ $\min \left(T_{2 v}(C), D_{2 v}(C)\right)$. Now it is easily verified that the clustering problem $(\mathcal{X}, \mathcal{L}, I, \tau, D, \mathcal{G})$ satisfies $P_{3}, P_{5}$ and $P_{6}$. Since this is a cluster analysis problem, it also satisfies $P_{1}, P_{2}$, and $P_{4}$.

We reduce the NP-hard graph $k$-colorability problem (Garey $\&$ Johnson, 1979) to the problem of finding a best solution among all clusterings having at most $k$ clusters for the problem $(\mathcal{X}, \mathcal{L}, I, \tau, \mathcal{D}, \mathcal{G})$. For each $k \geq 3$, the graph $k$-colorability problem is to determine whether there is a coloring of the vertices of a graph using at most $k$ colors, so that no two adjacent vertices have the same color. Given a graph $\mathcal{A}=(V, E)$ with $v$ vertices, we form an instance $\left(X_{2 v}, L_{2 v}, I_{2 v}, T_{2 v}, D_{2 v}, G_{2 v}, S\right)$ of the clustering problem above by letting the set $S \subset X_{2 v}$ to be clustered be exactly those elements of $X_{2 v}$ that represent vertices $V$ of the graph $A$ with adjacency information given by $E$. A simple argument shows that the graph $\mathcal{A}$ is $k$-colorable iff there is a clustering $C$ for this instance with at most $k$ clusters such that $G_{2 v}(C)=1$. (The clusters consist of representations of vertices to be colored with the same color.) Otherwise, any clustering for this instance 
with at most $k$ clusters has $G_{2 v}(C)=0$. It follows that for each $k$, any algorithm for solving $(\mathcal{X}, \mathcal{L}, I, \mathcal{T}, D, \mathcal{G})$ by finding the best clustering with at most $k$ clusters can be used to solve the graph $k$-colorability problem. We have thus proved

Theorem 3 For all $k \geq 3$, there are clustering problems $(\mathcal{X}, \mathcal{L}, I, \tau, \mathcal{D}$, G) satisfying properties $P_{1}$ through $P_{6}$ for which, unless $P=N P$, there exists no polynomial-time algorithm for finding a best clustering among all clusterings with at most $k$ clusters for every instance $\left(X_{n}, L_{n}, I_{n}, T_{n}, D_{n}\right.$, $\left.G_{n}, S\right)$.

\subsection{Variants of $T$ and $D$}

Although Theorem 1 shows that only very general assumptions on the functions $\mathcal{G}$ are needed, the results apply only when the functions $T$ satisfy property $P_{5}$ and the functions $D$ satisfy property $P_{3}$. Measures of tightness such as "density" of individual clusters allow the tightness to increase as a clustering is generalized, since "sparse" clusters may become "dense" when new points are added. Thus property $P_{5}$ is violated for this type of measure. Similarly, if the distance functions $\left\{D_{n}\right\}$ are defined as the maximum intercluster distance $d_{n}$, property $P_{3}$ is no longer satisfied. Below we show that (assuming $P \neq N P$ ) $P_{5}$ is necessary in the sense that properties $P_{1}, P_{2}, P_{3}, P_{4}$, and $P_{6}$ alone are not sufficient for a clustering problem to be solvable in polynomial time. We conclude by observing that if the functions $\left\{D_{n}\right\}$ are in fact the maximum intercluster distance, then the clustering problem is trivial (assuming $P_{5}$ still holds).

Theorem 4 There is a clustering problem $(\mathcal{X}, \mathcal{L}, I, T, \mathcal{D}, \mathcal{G})$ satisfying $P_{1}, P_{2}, P_{3}, P_{4}$, and $P_{6}$ that is not solvable in polynomial time unless $P=$ NP.

PROOF: We need only reduce INDEPENDENT SET, an $N P$-hard problem (Garey \& Johnson, 1979), to a cluster analysis problem satisfying properties $P_{3}$ and $P_{6}$. An instance of INDEPENDENT SET is a graph $A=(V, E)$ and a positive integer $k \leq|V|$. The problem is to determine if $A$ contains an independent set of size $k$ or more, i.e., a subset $V^{\prime} \subseteq V$ such that $\left|V^{\prime}\right| \geq k$ and such that no two vertices of $V^{\prime}$ are joined by an edge in $E$.

Let the clustering problem $(\mathcal{X}, \mathcal{L}, I, \tau, \mathcal{D}, \mathcal{G})$ be defined as in the proof of Theorem 3, except that $T_{2 v}$ has the modified definition given by: $T_{2 v}(C)$ $=0$ if the union of the clusters in $C$ is not a finite subset of $X_{2 v}$ representing all of the vertices of some undirected graph with $v$ vertices, or if there is a cluster $c \in C$ such the representations of two vertices that are adjacent in the represented graph are both contained in $c . T_{2 v}(C)=$ the number of elements in the largest cluster of $C$ otherwise. 
$(\mathcal{X}, \mathcal{L}, I, \tau, \mathcal{D}, \mathcal{G})$ satisfies properties $P_{1}, P_{2}, P_{3}, P_{4}$, and $P_{6}$, since only the definition of $\tau$ has been changed from the proof of Theorem 3, and property $P_{5}$ has been dropped. Also note that for each $C, D_{2 v}(C)=2 v \geq$ $T_{2 v}(C)$, so $G_{2 v}(C)=T_{2 v}(C)$. It is now easily shown that a graph $A$ has an independent set of size $k$ iff the instance $\left(X_{2 v}, L_{2 v}, I_{2 v}, T_{2 v}, D_{2 v}, G_{2 v}, S\right)$, with $S$ representing the graph $A$, has a solution $C$ with $G_{2 v}(C)=k$.

Finally, suppose that we modify part $(a)$ of property $P_{3}$, so that $D_{n}$ is now the maximum inter-cluster distance $d_{n}\left(c, c^{\prime}\right)$ among all clusters $c, c^{\prime} \in C$, where $d_{n}$ satisfies part $(b)$ of property $P_{3}$. Then any clustering problem $(\chi, \mathcal{L}, I, \tau, D, \mathcal{G})$ satisfying properties $P_{2}, P_{5}, P_{6}$, and this modified definition of $P_{3}$, is trivially solvable: the clustering given by $C_{1}$ in algorithm $A$ must be a best clustering, since it is a specialization of every best clustering; tightness cannot increase under generalization, nor can distance, by the modified property $P_{3}$. Thus $G_{n}$ cannot increase either.

\section{Conclusion}

In this paper we have examined the computational characteristics of an agglomerative clustering method. We can summarize the main results as follows:

- The agglomerative algorithm will find a best (conceptual) clustering of a set of points if the similarity measure (for clusterings) and the dissimilarity (between clusters) are monotone with respect to generalization, the objective function is monotone with respect to similarity and dissimilarity, and the language is tractable. In this case, "tractable" means that the clusterings of the language are not too large, that it is possible to efficiently determine whether a point is in a cluster, and that there exists (and it is possible to find) the most specific clustering in the language satisfying certain conditions. The "identity" language for cluster analysis trivially has these properties.

- Under these same conditions, the agglomerative algorithm will find a best clustering with at least $k$ clusters for any fixed $k$ less than the size of the sample set being clustered.

- Finding the best clustering with at most $k$ clusters is NP-hard under these conditions.

- If the measure of similarity is not monotone with respect to generalization, then finding an optimal clustering is NP-hard, even if the other monotone properties and the language properties are satisfied.

These results have several interesting implications. First, the agglomerative algorithm is more widely applicable than one would expect from such a simple technique. Under straightforward and intuitively natural 
conditions on the metric, the objective function, and the cluster description language, it finds a best clustering in polynomial time. The language restrictions are satisfied by conjunctive, attribute-based languages, including those using internal disjunction. They also apply to several interesting geometric languages. They do not hold for the existentially-quantified conjunctive predicate calculus statements that are sometimes used to represent structured objects (Stepp, 1987a; Larson, 1977).

Finally, as one would expect, it seems that finding a best clustering with a given number of clusters is hard. The implication is that clustering algorithms which try to find a best clustering of a certain size must be content with sub-optimal results. It also confirms the intuition that heuristic techniques and domain knowledge are probably necessary to produce good solutions.

We would like to extend the results to metrics that, for example, include notions such as density or average similarity over clusters. Additionally, it would be useful to be able to weaken the restrictions on distance (for a clustering) so that it need not be the minimum inter-cluster distance.

One problem we have not addressed here is the notion of predictive clustering, along lines of learnability as described by Valiant (1984) and Blumer, Ehrenfeucht, Haussler, and Warmuth (1986). (See also Kearns, $\mathrm{Li}$, Pitt, \& Valiant, 1987). The idea is to develop a clustering that is "good" for an entire space $X$ (under an unspecified probability distribution), given only randomly generated points from $X$. We have definitions that seem suitable for this problem, and we have some preliminary results indicating that this problem is significantly more difficult than nonpredictive clustering. These results may be presented in a future paper.

\section{Acknowledgements}

We are grateful to the referee for two thorough reviews and for simplifying the proof of Theorem 1 while extending its scope. L. Pitt was supported by the Department of Computer Science, University of Illinois at Urbana-Champaign, and R. E. Reinke was supported in part by the National Science Foundation under Grant IST-85-11170 and in part by the Office of Naval Research under Contract N00014-82-K-0186. 


\section{References}

Anderberg, M. (1973). Cluster analysis for applications. New York: Academic Press.

Blumer, A., Ehrenfeucht, A., Haussler, D., \& Warmuth, M. (1986). Classifying learnable geometric concepts with the Vapnik-Chervonenkis dimension. Proceedings of the Eighteenth Annual ACM Symposium on Theory of Computing (pp. 273-282). Berkeley, CA: The Association for Computing Machinery.

Carbonell, J. G., Michalski, R. S., \& Mitchell, T. M. (1983). An overview of machine learning. In R. S. Michalski, J. G. Carbonell, \& T. M. Mitchell (Eds.), Machine learning: An artificial intelligence approach. Los Altos. CA: Morgan Kaufmann.

Cohen, P. R., \& Feigenbaum, E. A. (Eds.). (1982). The handbook of artificial intelligence, Los Altos, CA: Morgan Kaufmann.

Duda, R., \& Hart, P. (1973). Pattern classification and scene analysis. New York: John Wiley.

Edelsbrunner, H. (1987). Algorithms in combinatorial geometry. In W. Brauer, G. Rozenberg, A. Salomaa (Eds.), Monographs in theoretical computer science (Vol. 10). Heidelberg: Springer-Verlag.

Everitt, B. (1980). Cluster analysis. London: Heinemann Educational Books.

Fisher, D. (1985). A proposed method of conceptual clustering for structured and decomposable objects. Proceedings of the Third International Machine Learning Workshop (pp. 38-40). Skytop, PA.

Fisher, D. (1987). Improving inference through conceptual clustering. Proceedings of the Sixth National Conference on Artificial Intelligence (pp. 461-465). Seattle, WA: Morgan Kaufmann.

Fisher, D., \& Langley, P. (1985). Approaches to conceptual clustering. Proceedings of the Ninth International Joint Conference on Artificial Intelligence (pp. 691 697). Los Angeles, CA: Morgan Kaufmann.

Garey, M., \& Johnson, D. (1979). Computers and intractability: A guide to the theory of NP-completeness. San Francisco, CA: W. H. Freeman.

Hartigan, J. (1975). Cluster algorithms. New York: John Wiley.

Kearns, M., Li, M., Pitt, L., \& Valiant, L. G. (1987). Recent results on Boolean concept learning. Proceedings of the Fourth International Workshop on Machine Learning (pp. 337-352). Irvine, CA: Morgan Kaufmann.

Larson, J. (1977). Inductive inference in the variable-valued predicate logic system VL21: Methodology and computer implementation. Doctoral dissertation, Department of Computer Science, University of Illinois. Urbana.

Lebowitz, M. (1983). Generalization from natural language text. Cognitive Science, $7,1-40$.

Michalski, R. S. (1980). Knowledge acquisition through conceptual clustering: A theoretical framework and an algorithm for partitioning data into conjunctive concepts. International Journal of Policy Analysis and Information Systems, 4, 219-243. 
Michalski, R. S. (1983). A theory and methodology of inductive learning. In R. S. Michalski, J. G. Carbonell, \& T. M. Mitchell (Eds.), Machine learning: An artificial intelligence approach. Los Altos, CA: Morgan Kaufmann.

Michalski, R. S., \& Stepp, R. E. (1983). Learning from observation: Conceptual clustering. In R. S. Michalski, J. G. Carbonell, \& T. M. Mitchell (Eds.), Machine learning: An artificial intelligence approach. Los Altos, CA: Morgan Kaufmann.

Mogenson, B. (1987). Goal-oriented conceptual clustering: The classifying attribute approach. Master's thesis, Department of Electrical and Computer Engineering, University of Illinois, Urbana.

Rendell, L. A. (1983). Toward a unified approach for conceptual knowledge acquisition. AI Magazine, 4, 19-27.

Romesburg, H. (1984). Cluster analysis for researchers. Belmont, CA: Lifetime Learning.

Stepp, R. (1984). Conjunctive conceptual clustering: A methodology and experimentation. Doctoral dissertation, Department of Computer Science, University of Illinois, Urbana.

Stepp, R. (1987a). Machine learning from structured objects. Proceedings of the Fourth International Workshop on Machine Learning (pp. 353-363). Irvine, CA: Morgan Kaufmann.

Stepp, R. (1987b). Concepts in conceptual clustering. Proceedings of the Tenth International Joint Conference on Artificial Intelligence (pp. 211-213). Milan, Italy: Morgan Kaufmann.

Stepp, R., \& Michalski, R. S. (1986). Conceptual clustering: Inventing goaloriented classifications of structured objects. In R. S. Michalski, J. G. Carbonell, \& T. M. Mitchell (Eds.), Machine learning: An artificial intelligence approach (Vol. 2). Los Altos, CA: Morgan Kaufmann.

Valiant, L. G. (1984). A theory of the learnable. Communications of the ACM, 27, 1134-1142. 